\section{Câncer cérvico-uterino como condição marcadora: uma proposta de avaliação da atenção básica}

\author{
Cervical cancer as a tracer condition: a proposal \\ for evaluation of primary health care
}

\footnotetext{
${ }^{1}$ Escola Nacional de Saúde Pública Sergio Arouca, Fundação Oswaldo Cruz, Rio de Janeiro, Brasil. 2 Hospital Universitário Gafrée e Guinle, Universidade Federal do Estado do Rio de Janeiro, Rio de Janeiro, Brasil.

Correspondência C. M. S. Bottari Departamento de Administração e Planejamento em Saúde, Escola Nacional de Saúde Pública Sergio Arouca, Fundação Oswaldo Cruz. Rua Leopoldo Bulhões 1480, sala 727 , Rio de Janeiro, $R J$ 21041-210, Brasil.

clairemoraes@gmail.com clairemoraes@hotmail.com
}

\begin{abstract}
The use of tracer conditions as an evaluative technique suggests the possibility of inferring the quality of health care and setting evaluation standards for programmed actions. As a government strategy, primary health care is a key element for reorganization of the Brazilian health care model. This study analyzes the use of cervical cancer as a tracer condition for assessing primary care. Based on the results from questions on measures to control the disease from the perspective of health professionals and cervical cancer patients, we constructed a matrix of indicators relating primary care attributes to process/results indicators. The analytical plan to validate the results used triangulation of methods, associating data from qualitative and quantitative approaches, in addition to combining and cross-analyzing the various actors' points of view. The results suggest that cervical cancer is an excellent tracer condition for primary care in general, based on the similarity of such concepts as accessibility, coverage, comprehensiveness, technical and scientific quality, and effectiveness.
\end{abstract}

Primary Health Care; Heath Evaluation; Uterine Neoplasms
Clarissa Moraes de Sousa Bottari 1,2

Miguel Murat Vasconcellos 1

Maria Helena Magalhães de Mendonça ${ }^{1}$

\section{Introdução}

Segundo Novaes 1, a produção de conhecimentos voltados para o foco avaliativo ainda não é tão expressiva no Brasil, como se evidencia pelo pequeno número de publicações sobre o tema em periódicos científicos brasileiros. Devido a tal panorama e a crescente necessidade da avaliação dos serviços, impõe-se a produção cada vez maior de trabalhos sobre avaliação de serviços, de rede e serviços, e a experimentação de novas metodologias que facilitem a realização do processo avaliativo nas unidades assistenciais e nas esferas gestoras.

A utilização de condições denominadas marcadoras, ou traçadoras, pode ser uma forma de avaliar a qualidade da assistência à saúde 2 . Autores como Kessner et al. ${ }^{3}$, Travassos 4 e Penna 5 referem a utilização dessa técnica, fundada na idéia de que a partir da avaliação da assistência prestada a um conjunto de determinadas condições ou patologias, pode-se inferir a qualidade da atenção à saúde em geral, incluindo-se aí a resolutividade, uso adequado de exames complementares, oportunidade das ações, acesso a medicamentos ou a níveis mais complexos do sistema de saúde. Para Hartz \& Contandriopoulos 6, o desenvolvimento de método avaliativo pautado no uso de condição marcadora facilita a definição dos focos nas avaliações das ações programáticas de saúde e aumenta o potencial de utilidade dos resultados das pesquisas. 
Penna 5 afirma que as condições marcadoras devem ser escolhidas entre aquelas para as quais existam "programas" já consolidados, cujas normas de assistência conseguiram uma aprovação consensual por parte dos profissionais de saúde, e que se sustentam por definição prévia acerca dos recursos e procedimentos adequados a cada nível de atenção para a implementação de atividades.

O câncer cérvico-uterino possui etiologia, diagnóstico, terapêutica e prevenção bem definidos, o que significa que existe um conhecimento científico aprofundado sobre os fatores causais, formas de detecção e tratamento da doença. Também é objeto de um programa nacional estruturado para seu controle. A doença possui evolução lenta e seu tratamento é conhecido e eficaz, principalmente nas lesões precursoras. Nesses casos, o tratamento cirúrgico é curativo em $100 \% 7$. As características biológicas do câncer do colo do útero e a existência de um método simples, barato, seguro e aceitável pela população feminina para a sua detecção precoce, o exame citopatológico (Papanicolaou), permitem que seu potencial de prevenção e cura seja elevado (Viva Mulher - Programa Nacional de Controle do Câncer do Colo Útero e de Mama http://www.inca.gov.br/prevencao/programas/ viva_mulher/documentos/vivamulher_faseI. pdf, acessado em 20/Jul/2005).

Segundo Bicalho \& Aleixo ${ }^{8}$, o câncer de colo de útero detém o terceiro lugar em incidência e o quarto em mortalidade no Brasil. Em 2000 apresentou 17.251 casos novos e 3.606 mortes. A estimativa do Instituto Nacional de Câncer (INCA) para 2006, no Brasil, é que o câncer de colo uterino ocuparia o segundo lugar em incidência em mulheres, com 19 mil casos novos (risco estimado de vinte casos por 100 mil mulheres) 9 .

Kessner et al. 3 selecionam o câncer cérvicouterino como condição marcadora com base em alguns critérios. Dentre eles estabelecem que a condição deve permitir avaliar o cuidado ambulatorial prestado. Samico et al. 10 também definem o câncer de colo uterino como marcador, pois é evento prevalente em nosso meio e pode ser utilizado como elemento desencadeador de um debate importante entre profissionais e usuários, visando a entendê-los enquanto integrantes de um processo que se apóia no modo de inserção social das pessoas.

Considera-se que o programa de controle do câncer cérvico-uterino envolve todos os níveis de atenção no seu cuidado, com ênfase nas ações preventivas e de detecção precoce, que se concentram na Atenção Básica ampliada, como definida na Norma Operacional de Atenção à Saúde (NOAS 2001).
As ações preventivas de educação em saúde, detecção por meio da colpocitologia e encaminhamento para tratamento em níveis de maior complexidade são de responsabilidade da Atenção Básica. Mesmo após a cura, esta ainda permanece responsável pelo acompanhamento das usuárias, mantendo as ações de sua competência, e assim prevenindo recidivas.

O objetivo deste artigo é refletir sobre as possibilidades da condição marcadora câncer cérvico-uterino como mecanismo de avaliação da Atenção Básica enquanto estratégia do Sistema Único de Saúde (SUS). Inserido no contexto da pesquisa Desenvolvimento de Estudo de Linhas de Base nos Municípios Selecionados do Projeto de Expansão e Consolidação do Saúde da Família - Região Sudeste 2 (ELB-PROESF), cuja proposta foi avaliar situações iniciais que condicionam o processo de implementação da Atenção Básica buscando orientar sua organização, foram analisadas triangulações, cruzamentos e combinações entre indicadores qualitativos e quantitativos classificados a partir de temas, processos e resultados.

\section{Materiais e métodos}

O presente trabalho insere-se no estudo avaliativo realizado pelo ELB-PROESF, pesquisa de natureza quali-quantitativa que abrangeu municípios com população acima de 100 mil habitantes no Estado do Rio de Janeiro ${ }^{11}$. A avaliação da Atenção Básica realizada por meio do ELB-PROESF produziu resultados acerca das diversas dimensões relevantes para esta esfera de atenção. Selecionamos para este estudo apenas os resultados produzidos pelo ELB-PROESF para um dos municípios selecionados para estudo de caso, denominado Município 3. Trata-se de um município de grande porte, com população de $450 \mathrm{mil}$ habitantes. Possui cinqüenta unidades públicas de Atenção Básica e uma cobertura de 65,8\% do município pelo Programa Médico de Família.

As fontes de dados do estudo foram os resultados obtidos por diferentes instrumentos construídos pelo ELB-PROESF para abordar diversas dimensões: contexto político institucional, organização da Atenção Básica e trajetória do Programa Saúde da Família (PSF) no SUS municipal, integralidade do cuidado e desempenho da Atenção Básica - através de cinco instrumentos voltados para os atores envolvidos no processo de implementação do PROESF. Esses atores representam distintos interesses quanto à assistência prestada na Atenção Básica - usuários, profissionais de saúde, gerentes de estabelecimentos de saúde e gestores de saúde. 
O câncer de colo uterino, enquanto condição marcadora, foi tratado em todos os instrumentos desenvolvidos, visando a articular as dimensões em seus resultados, comparar situações e percepções relativas à assistência de mulheres em face deste evento. Os instrumentos utilizados foram:

- Questionário aplicado junto à equipe gestora da Secretaria Municipal de Saúde (IG), que levantou informações relativas ao esquema de referência para realização do exame colpocitológico e encaminhamento de mulheres com resultado alterado.

- Inquérito de estabelecimentos (IE) de Atenção Básica de saúde com e sem PSF, igualmente visou a apreender sobre o esquema de referência, capacidade de gestão deste esquema e as práticas do processo de trabalho nestas unidades refletidas nas condições de registro dos atendimentos feitos em mulheres acima de 19 anos, especialmente referentes à realização do preventivo ginecológico e presença do registro do resultado do mesmo;

- Grupos focais (GF) com mulheres com exames alterados ou portadoras de câncer de colo e profissionais de saúde da Atenção Básica e de serviços de referência, tratando do acesso e cuidado à saúde da mulher;

- Inquérito domiciliar (ID) em que foi perguntado às mulheres sobre a época de realização e recebimento do resultado do último preventivo de câncer de colo uterino;

- Estudo ecológico (EC), demonstrando as variações da taxa de mortalidade por câncer cérvico-uterino nos municípios estudados em anos selecionados.

\section{Plano e análise dos dados}

O plano de análise utilizado para validar a avaliação pela condição marcadora faz uso da triangulação 12 de métodos e técnicas de investigação, associando resultados provenientes das abordagens qualitativa (GF) e quantitativa (IG, IE e ID e EC), além de combinar e cruzar diversos pontos de vista apreendidos dos atores envolvidos. $\mathrm{O}$ pressuposto é que o câncer cérvico-uterino é um bom marcador da assistência ofertada na Atenção Básica, ou seja, a partir desta condição marcadora pode-se analisar o funcionamento dos seus serviços, bem como a percepção de usuárias e profissionais de saúde quanto à prevenção, controle da causa da doença, seu diagnóstico precoce, sua referência adequada e tratamento. Os resultados encontrados usando-se a condição marcadora aplicariam-se à Atenção Básica como um todo.

Para este estudo levantou-se os principais temas abordados na pesquisa ELB-PROESF e os indicadores qualitativos e quantitativos que com eles se relacionam, segundo o instrumento do qual foi extraído. Esta classificação compõe uma matriz apresentada na Tabela 1. Tal abordagem baseia-se em Lopes et al. ${ }^{2}$, que propõem um modelo avaliativo com base na elaboração de uma matriz com diferentes níveis de análise como mecanismo para estruturar os resultados.

Para avaliar o uso da condição marcadora, selecionou-se o estudo de caso do Município 3 realizado na pesquisa ELB-PROESF. Os temas abordados são: acesso; contexto político institucional; organização da atenção básica e PSF; recursos humanos; perfil dos serviços e capacidade instalada; integralidade e práticas de atenção à saúde; condições de registros nos estabelecimentos; mortalidade.

Kessner et al. 3 indicam que o uso da condição marcadora permite avaliar processo e resultado da atenção prestada. Donabedian 13 define estrutura como categoria que inclui os recursos materiais, humanos e a estrutura organizacional; processo como categoria que inclui todo o cuidado, desde a sua busca e apreensão pelo usuário do serviço de saúde até o atendimento prestado, incluindo diagnóstico, prescrição e implementação do tratamento; e resultado como os efeitos do cuidado na situação de saúde dos pacientes e população. Ambos se relacionam diretamente, ou seja, quando o processo está bem estruturado terá como conseqüência um bom resultado.

Após a primeira estruturação temática dos indicadores foi elaborada uma classificação em "Indicadores de Estrutura”, "Indicadores de Processo" e "Indicadores de Resultado” para então realizar um segundo agrupamento por subtemas. Assim, foram construídas as Tabelas 2, 3 e 4. O primeiro refere-se à avaliação de estrutura, o segundo à avaliação de processo e o terceiro à de resultados. Os subtemas referem-se a atributos de serviços, programas ou sistema de saúde (neste caso a Atenção Básica) que ao serem avaliados apuram a análise e contribuem para o aprofundamento do processo que define o foco da avaliação ${ }^{14}$. Os atributos selecionados baseiam-se no referencial de Starfield 15 e Silva ${ }^{14}$ e são descritos a seguir:

- Acessibilidade: relaciona-se a tudo que se refere ao acesso da população aos serviços (localização da unidade, dia e horário de atendimento, acesso à livre demanda) 16. Também é definida como a relação dos recursos de poder dos usuários (econômicos, sociais ou culturais) e os obstáculos existentes (geográficos, organizacionais ou econômicos) 15. Os indicadores classificados neste subtema refletem o acesso da população feminina à Atenção Básica, bem como à coleta da colpocitologia; 
Matriz de indicadores avaliativos classificados por temas e fonte de dados.

\begin{tabular}{|c|c|c|}
\hline Tema & Indicador & Fonte/Instrumento \\
\hline \multirow[t]{5}{*}{ Acesso } & Realização do último exame ginecológico nos últimos 3 anos & ID \\
\hline & Realização do exame preventivo no último exame ginecológico & ID \\
\hline & Recebimento do resultado da última colpocitologia em menos de 1 mês & ID \\
\hline & Adscrição da clientela e acessibilidade & GF \\
\hline & Cobertura dos programas & GF \\
\hline \multirow[t]{2}{*}{ Contexto político institucional } & Desempenho do sistema e situação de saúde & GF \\
\hline & Intersetorialidade & GF \\
\hline \multirow[t]{17}{*}{ Organização da Atenção Básica e PSF } & Estabelecimentos segundo trabalho com clientela adscrita & IE \\
\hline & Adscrição da clientela e acessibilidade & GF \\
\hline & Capacidade de informar colpocitologias realizadas em 2003 & IE \\
\hline & Capacidade de informar colpocitologias realizadas em 2004 & IE \\
\hline & Capacidade de informar colpocitologias alteradas em 2003 & IE \\
\hline & Capacidade de informar colpocitologias alteradas em 2004 & IE \\
\hline & Acesso a serviços de referência para colpocitologia & IE \\
\hline & Forma de acesso em caso de colpocitologia alterada & IE \\
\hline & (laboratório informa resultado ao Centro de Saúde de origem, que & \\
\hline & possui referência formal para agendamento de consulta com especialista) & \\
\hline & Percepção sobre o modelo de atenção adotado na Atenção Básica/PSF & GF \\
\hline & Percepção sobre organização e hierarquização da rede/integração dos serviços & GF \\
\hline & Laboratório de patologia de referência para análise de colpocitologia & IE \\
\hline & Serviços de referência para colpocitologia no município & IG \\
\hline & Serviços de referência para colpocitologia fora do município & IG \\
\hline & Serviços de referência para colposcopia no município & IG \\
\hline & Serviços de referência para colposcopia fora do município & IG \\
\hline Recursos humanos & Proporção de vínculos de gineco-obstetras por total de médicos & IE \\
\hline \multirow[t]{2}{*}{ Perfil dos serviços e capacidade instalada } & Estabelecimentos com pronto atendimento em ginecologia & IE \\
\hline & Estabelecimentos com ambulatório em ginecologia & IE \\
\hline \multirow[t]{4}{*}{ Integralidades práticas de atenção à saúde } & Estabelecimentos com coleta de citopatológico & IE \\
\hline & Percepção sobre saúde & GF \\
\hline & Práticas adotadas/qualidade do cuidado com a saúde das pessoas & GF \\
\hline & Informação/Comunicação & GF \\
\hline \multirow[t]{2}{*}{ Condições de registros nos estabelecimentos } & Prontuários com registro de realização de colpocitologia & AA \\
\hline & Prontuários com presença do resultado do exame colpocitológico & AA \\
\hline Mortalidade & Taxa de mortalidade por câncer de colo uterino em 2002 & EC \\
\hline
\end{tabular}

ID: inquérito domiciliar; GF: grupo focal; IE: inquérito nos estabelecimentos; IG: inquérito com o gestor; AA: análise dos atendimentos; EC: estudo ecológico; PSF: Programa Saúde da Família.

- Cobertura/Adscrição: tem duas compreensões, a saber: potencial - quando se mede recursos, sejam materiais ou humanos, para a realização da ação em saúde; e real - proporção da população que de fato utilizou um serviço ou se beneficiou de determinada intervenção 15 . A adscrição da clientela é considerada como forma de reorganização gerencial e operacional da Atenção Básica. O Ministério da Saúde recomenda que a unidade básica de saúde se responsabilize por um número compatível de pessoas que residem na área próxima, ou seja, devem trabalhar com definição de área de abrangência e de população sob sua responsabilidade, que deve ser cadastrada e vinculada à unidade 16. Portanto, a partir da adscrição é possível prever a população que deverá ser coberta por uma determinada unidade de saúde. Os 
Matriz de indicadores de estrutura.

\begin{tabular}{ll}
\hline Subtema & \multicolumn{1}{c}{ Indicador } \\
\hline \multirow{2}{*}{ Acessibilidade } & Proporção de vínculos de gineco-obstetras por total de médicos \\
& Estabelecimentos com pronto atendimento em ginecologia \\
& Estabelecimentos com ambulatório em ginecologia \\
& Laboratório de patologia de referência para análise de colpocitologia \\
Integralidade & Forma de acesso em caso de colpocitologia alterada (laboratório informa resultado ao \\
& Centro de Saúde de origem, que possui referência formal para agendamento) \\
& Serviços de referência para colpocitologia no município \\
& Serviços de referência para colposcopia no município \\
$93,3 \%$
\end{tabular}

Tabela 3

Matriz de indicadores de processo.

\begin{tabular}{|c|c|c|}
\hline Subtema & Indicador & Resultado \\
\hline \multirow[t]{4}{*}{ Acessibilidade } & Realização do último exame ginecológico nos últimos 3 anos & $88,3 \%$ \\
\hline & Realização do exame preventivo no último exame ginecológico & $93,3 \%$ \\
\hline & Recebimento do resultado da última colpocitologia em menos de 1 mês & $73,5 \%$ \\
\hline & Adscrição da clientela e acessibilidade & $\begin{array}{l}\text { Coleta de preventivo em diversos locais, } \\
\text { dificultando seguimento da população adscrita }\end{array}$ \\
\hline \multirow[t]{6}{*}{ Cobertura/Adscrição } & Cobertura dos Programas & Atendimento à população de municípios vizinhos \\
\hline & Estabelecimentos segundo trabalho com clientela adscrita & $93,3 \%$ \\
\hline & Capacidade de informar colpocitologias realizadas em 2003 & $20,0 \%$ \\
\hline & Capacidade de informar colpocitologias realizadas em 2004 & $26,7 \%$ \\
\hline & Capacidade de informar colpocitologias alteradas em 2003 & $16,7 \%$ \\
\hline & Capacidade de informar colpocitologias alteradas em 2004 & $20,0 \%$ \\
\hline \multirow[t]{8}{*}{ Integralidade } & Intersetorialidade & Iniciativa inconstante de trabalho intersetorial \\
\hline & Percepção sobre o modelo de atenção adotado na Atenção Básica/PSF & $\begin{array}{l}\text { Usuárias identificam a busca ativa e possuem } \\
\text { vínculo com a unidade }\end{array}$ \\
\hline & $\begin{array}{l}\text { Percepção sobre organização e hierarquização da rede/ } \\
\text { integração de serviços }\end{array}$ & $\begin{array}{l}\text { Reconhecimento do sistema de saúde em rede, } \\
\text { porém com dificuldades de funcionamento }\end{array}$ \\
\hline & Estabelecimentos com coleta de citopatológico & $93,3 \%$ \\
\hline & Percepção sobre saúde & Conceito ampliado de saúde \\
\hline & Práticas adotadas/qualidade do cuidado com a saúde das pessoas & Promoção à saúde individualizada \\
\hline & Acesso a serviços de referência para colpocitologia & $93,3 \%$ \\
\hline & Desempenho do sistema e situação de saúde & $\begin{array}{l}\text { Dificuldades para referência; inexistência } \\
\text { de contra-referência }\end{array}$ \\
\hline Continuidade/Qualidade & Prontuários com registro de realização de colpocitologia & $54,7 \%$ \\
\hline Técnico-científica & Prontuários com presença do resultado do exame colpocitológico & $36,3 \%$ \\
\hline
\end{tabular}

indicadores de cobertura medem a capacidade de programação e gerenciamento das unidades, incluindo o retorno de resultados de exames à usuária;

- Integralidade: é um dos princípios do SUS e compreende o atendimento integral, ou seja, de todas as necessidades de saúde apresentadas pelo indivíduo, com ênfase na prevenção e sem prejuízo da assistência ${ }^{17}$. Para Starfield 15 , é entendida como a organização da Atenção Básica para que o usuário receba todos os tipos de serviços de atenção à saúde, mesmo aqueles que 
Tabela 4

Matriz de indicadores de resultado.

\begin{tabular}{ll}
\hline Subtema & Indicador \\
\hline Efetividade & Taxa de mortalidade por câncer de colo uterino \\
\hline
\end{tabular}

são oferecidos fora desta unidade, neste caso por meio de encaminhamentos. Mattos 17, compreende integralidade além do seu sentido de garantia de acesso a todos os níveis do sistema de saúde, partindo do princípio de que envolve a interação dos profissionais com os usuários dos serviços de saúde, tanto no atendimento às suas demandas (experiência individual de sofrimento) quanto nas ações preventivas (antecipação à experiência individual de sofrimento). Os indicadores de integralidade permitem avaliar a organização dos serviços na garantia de atendimento para todas as necessidades apresentadas pelas usuárias, desde ações preventivas, como a oferta do exame colpocitológico, até o sistema de referência para níveis de maior complexidade. Os indicadores qualitativos tornam possível conhecer a percepção dos profissionais e usuárias a respeito da organização do sistema, assim como as práticas de saúde adotadas e a existência de ações intersetoriais para promoção à saúde, fundamentais para a efetiva integralidade do cuidado e alteração do modelo assistencial;

- Continuidade/Qualidade técnico-científica: a continuidade relaciona-se a arranjos do serviço para que a atenção oferecida apresente uma sucessão ininterrupta de eventos, incluindo mecanismos que caracterizam a continuidade da assistência, como o registro em prontuários $16 \mathrm{e}$ seguimento dos usuários (follow up). Qualidade técnico-científica corresponde a adequação das ações ao conhecimento técnico-científico vigente 15 . Os dois conceitos possuem relação direta. A análise do registro no prontuário permite avaliar a continuidade da assistência prestada, bem como identificar se a mesma ajusta-se ao conhecimento técnico-científico disponível;

- Efetividade: definida por autores como Donabedian (1990), Maynard (2003) e McDaid (2003) (citados por Silva ${ }^{14}$ ) como o efeito da intervenção em sistemas operacionais. A mortalidade pelo câncer de colo uterino em 2002 foi o indicador elaborado com base no EC para expressar este atributo.

Nas Tabelas 2 e 3 atribuem-se aos indicadores valores apreendidos para o Município 3 do ELBPROESF

\section{Resultados}

A avaliação dos atributos da Atenção Básica ocorreu por meio da perspectiva da triangulação, que conjugou dados qualitativos e quantitativos, e contou com a participação dos atores envolvidos - profissionais e usuárias - na produção destes resultados. Durante o processo avaliativo a condição marcadora esteve presente em todos os instrumentos utilizados. Os resultados obtidos mediante a avaliação, tendo a condição marcadora presente como pano de fundo, podem ser inferidos para a Atenção Básica em geral, como representado na Figura 1.

\section{Indicadores de estrutura}

O sistema de saúde no Município 3 apresentou a proporção de $20 \%$ de vínculos de gineco-obstetras por total de médicos (IG), enquanto nos serviços de saúde (IE) apurou-se que estes se organizam de forma diferenciada quanto ao atendimento, verificando que $66,7 \%$ dos estabelecimentos referiram possuir pronto atendimento em ginecologia e $43,3 \%$ terem oferta programada (ambulatório) de ginecologia.

A presença de serviço de referência para colpocitologia e de laboratório de patologia de referência para análise de colpocitologia foi relatada por 93,3\% dos estabelecimentos. O mesmo percentual de estabelecimentos referiu como forma de acesso em caso de colpocitologia alterada que o laboratório informa o resultado ao centro de saúde de origem, que possui referência formalizada para consulta com especialista.

Em relação à disponibilidade de referência para colpocitologia e colposcopia, o gestor do Município 3 informou que a referência existe para os dois tipos de serviço dentro do município. $\mathrm{O}$ serviço de referência concentra-se em uma única policlínica, que atende a todos os encaminhamentos de mulheres com colpocitologia alterada e realiza análise laboratorial de todas as lâminas de colpocitologia no município. 


\section{Indicadores de processo}

Quanto ao acesso ao atendimento na Atenção Básica, 88,3\% das mulheres investigadas no ID referiram realização de consulta ginecológica nos últimos três anos; destas, 93,3\% mencionaram a realização de colpocitologia no último exame ginecológico e 73,5\% o recebimento do resultado do exame em um mês.

Sobre a adscrição de clientela e acessibilidade foram identificadas pelos profissionais dificuldades para o acompanhamento das mulheres devido à realização do exame preventivo em diversos locais.

Quanto à acessibilidade à Atenção Básica com e sem PSF, seus profissionais identificaram dificuldades de acesso pela população ao serviço de saúde, dificuldade em avaliar o perfil das usuárias e busca de atendimento nas unidades pela população fora de área.

O relato de trabalho com população adscrita foi referido por $93,3 \%$ dos estabelecimentos. Em relação às colpocitologias realizadas, $20 \%$ das unidades foram capazes de informar colpocitologias realizadas em 2003, e 26,7\% em 2004; $16,7 \%$ informaram colpocitologias alteradas em 2003, e $20 \%$ em 2004.

No que se refere à intersetorialidade, os profissionais de saúde referiram iniciativa para o trabalho intersetorial, porém inconstante, ou seja, não há uma prática rotineira de atividades intersetoriais. Quando essas acontecem, é de forma esporádica.

Sobre a percepção do modelo de atenção adotado na Atenção Básica/PSF, foi identificada pelas usuárias a existência de busca ativa pelos profissionais da unidade. Referiram também possuir algum vínculo com a unidade, porém desconhecendo as atividades que ali ocorrem.

Em relação à percepção sobre a organização e hierarquização da rede/integração de serviços, os profissionais de saúde afirmaram que há absorção da demanda com sobrecarga de trabalho; identificaram o sistema de saúde em rede, porém com baixa troca de informações.

No que se refere às ações de saúde preconizadas nos programas de saúde coletiva, 93,3\% dos estabelecimentos afirmaram possuir coleta de colpocitologia.

As usuárias investigadas no GF perceberam saúde como ausência de doença, e mostraram ter conhecimento sobre o câncer de colo uterino. Os profissionais definiram saúde a partir de seu conceito ampliado. Esse grupo também demonstrou valorização da promoção à saúde, porém com limitação na sua prática (práticas adotadas/qualidade do cuidado com a saúde das pessoas).
Figura 1

Esquema demonstrativo dos resultados do estudo.

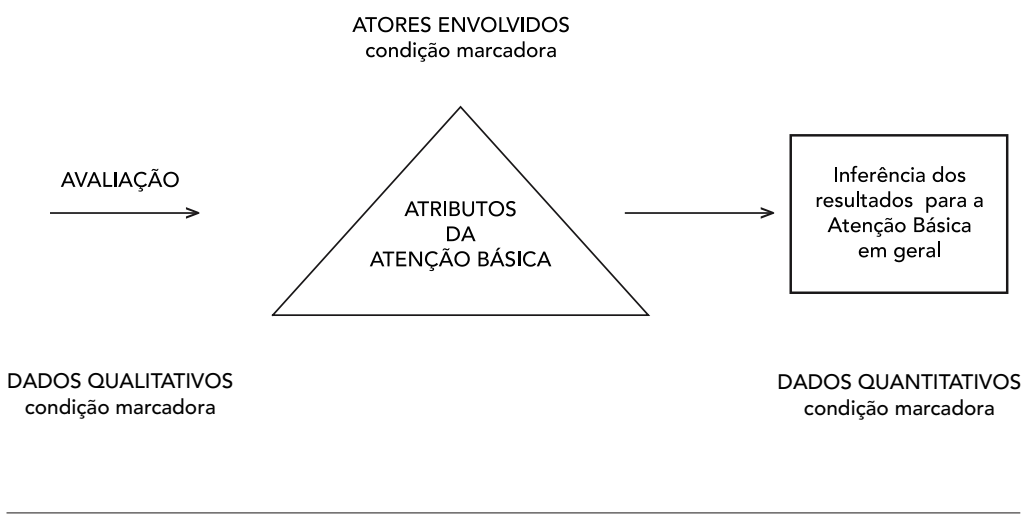

Foi identificada pelos profissionais de saúde a estrutura do sistema de saúde em rede, porém com limitações para a referência das usuárias devido ao número de vagas insuficiente. A contrareferência foi considerada inexistente.

$\mathrm{O}$ registro de realização de colpocitologia esteve presente em $54,7 \%$ dos atendimentos pesquisados em amostra estratificada dos atendimentos realizados na Atenção Básica no $\mathrm{Mu}$ nicípio 3 em 2004; a presença do resultado da colpocitologia no prontuário esteve registrada em apenas $36,3 \%$ dos atendimentos.

\section{Indicadores de resultado}

A taxa de mortalidade pela condição marcadora foi de 5,47 para 100 mil mulheres em 2002.

\section{Discussão}

$\mathrm{Na}$ análise que segue, os indicadores quantitativos e qualitativos de cada subtema são discutidos em conjunto; indica-se a complementaridade ou oposição dos resultados obtidos pelos indicadores que orientam as conclusões acerca da qualidade da Atenção Básica e a potencialidade do uso desta condição marcadora.

O percentual de usuárias que busca algum tipo de serviço de saúde para realização de consulta ginecológica é alto, assim como o de mulheres que realizaram o Papanicolau há menos de três anos e o percentual de mulheres que receberam o resultado do exame preventivo em menos de 
um mês, independentemente da natureza do prestador - público ou privado.

Segundo a Organização Mundial da Saúde (OMS), diversos estudos demonstram que a realização do preventivo ginecológico a cada 3 a 5 anos após um resultado negativo tem o mesmo efeito que a realização anual do exame por mulheres na faixa etária de 35 a 64 anos 18. O Ministério da Saúde recomenda que o tempo para realização do preventivo ginecológico seja de três anos 19 .

Em 2006, foi pactuado entre os gestores do SUS que a cobertura populacional pelo exame citopatológico cervical superior a $80 \%$ da população alvo seria considerado como padrão de referência 20 . O percentual de $93,3 \%$ de mulheres com colpocitologia realizada a menos de três anos denota boa cobertura populacional no Município 3 para o screening de câncer cérvicouterino.

No Canadá, autoridades governamentais recomendam como tempo ideal entre a coleta e a liberação do laudo citopatológico cerca de trinta dias 21 . Esse período permite garantir intervenção no tempo adequado, facilita a adesão e fortalece a credibilidade da mulher, além de possibilitar avaliar as etapas entre a coleta do material e a liberação do laudo.

Esse resultado bastante positivo nesse município quanto ao acesso e regularidade do exame preventivo pode ser contrastado com as falas dos profissionais de saúde da Atenção Básica. Para eles há dificuldades no acompanhamento das mulheres, que procuram a realização do exame preventivo em diversos locais, fazendo fluxos próprios que a adscrição de clientela e acessibilidade deveriam sanar. No grupo focal os profissionais da Atenção Básica em saúde da família afirmaram ter dificuldades para o acompanhamento da população adscrita devido à realização do preventivo em diversos lugares; as usuárias por sua vez relataram dificuldades na qualidade do atendimento, em especial, a demora do resultado dos exames (fala de uma usuária: “... quase seis meses, muito demorado e eu correndo atrás...”).

Os dados qualitativos permitiram detectar alguns pontos fracos para a acessibilidade. Os profissionais de saúde do serviço de referência identificaram que com a implantação da estratégia saúde da família houve uma melhoria no acesso, porém, a demanda de atendimento aumentou muito, gerando uma demora de até um mês para o agendamento de consultas. Afirmam que a própria população reconhece a falta de acesso.

Diante de uma demanda apontada pelos profissionais da Atenção Básica como "excessiva”, verificou-se uma inadequação da capacidade instalada para o atendimento às mulheres do município, com uma oferta de ambulatórios de ginecologia baixa $(43,3 \%$ das unidades investigadas, ou seja, menos da metade), assim como de pronto atendimento para a especialidade (66,7\% das unidades estudadas). Para buscar suprir essas dificuldades, os profissionais apontaram algumas alternativas para facilitar o acesso das mulheres ao serviço, tais como encaixes e oferta diária de vagas específicas para coleta de preventivo.

A análise dos dados quantitativos e qualitativos levanta como questão se as usuárias não estariam buscando atendimento em serviços privados.

A baixa capacidade de informar colpocitologias realizadas e alteradas em 2003 e 2004 nos estabelecimentos de saúde pode ser um fator explicativo dessa contradição. Apesar do percentual de estabelecimentos que referiu trabalhar com clientela adscrita ter sido alto, verifica-se que não utilizaram o indicador cobertura de colpocitologias alteradas na sua área de adscrição, sugerindo baixa capacidade de gerenciamento. Segundo os profissionais do serviço de referência existe dificuldade em avaliar o perfil das usuárias, saber quantas mulheres fazem o preventivo porque “... os laboratórios não repassam informações, tem lugares que fazem o preventivo muito barato, as clínicas de Igreja também fazem, as pessoas então não vão (...) no posto de saúde, daí que não temos os dados de incidência, de prevalência em relação à população que tem o preventivo em dia, só temos os dados das que chegam ao nosso serviço" (fala de um profissional de saúde).

O discurso apresentado pelo profissional do serviço de referência mostra uma visão focada no modelo médico-assistencial privatista, no qual a busca às unidades de saúde se dá a partir do surgimento de um problema de saúde. Como as usuárias conseguem realizar o preventivo ginecológico em locais privados, suprindo esta necessidade de saúde, não buscam as unidades públicas de saúde para mostrar o resultado. Embora exista um movimento em prol da mudança do modelo assistencial expressa na Norma Operacional Básica/96, que incentiva por meio de recursos financeiros estratégias como o PSF e ações de vigilância epidemiológica e sanitária, incorporando o modelo de "vigilância da saúde" 22, os profissionais ainda possuem uma visão da atenção à saúde no outro modelo. Não conhecem as responsabilidades assumidas pelos profissionais a partir da nova proposta de modelo assitencial, em que o PSF apresenta-se como eixo central de reorganização, realizando visitas domiciliares para acompanhamento da população adscrita e a busca ativa. 
O conhecimento acerca do número de colpocitologias realizadas no decorrer do ano, assim como o conhecimento do número de resultados alterados, são fundamentais para a programação de ações voltadas para o controle do câncer cérvico-uterino. É por meio da análise desses dados que a unidade poderá conhecer melhor a sua população, calcular a cobertura da população adscrita, reconhecendo os pontos chave a serem trabalhados, tais como educação em saúde ou captação de mulheres. Além disso, o conhecimento desses dados permite programar gastos com recursos materiais e humanos.

Embora desconheçam a cobertura, os profissionais da Atenção Básica informaram oferecer atendimento muito além da população adscrita. Relatam que atendem mulheres de municípios vizinhos, que forjam o endereço para conseguirem atendimento, fato identificado quando há necessidade de busca ativa da paciente devido a alterações em resultados de exames.

O sistema de saúde é percebido pelos profissionais da Atenção Básica como uma rede (relato de recebimento de pacientes pelo serviço de referência). Relatam que o município se caracteriza por ter uma boa troca entre os profissionais, facilitada algumas vezes pela proximidade (por exemplo, o laboratório de referência para colpocitologia do município que se situa no mesmo prédio do serviço de referência ambulatorial para câncer cérvico-uterino). Entretanto, relatam perceber que nos últimos tempos vem ocorrendo uma "impessoalidade", tornando este sentimento de pertencimento da rede, frágil.

A integralidade é aparentemente garantida pela presença de serviços de referência para colpocitologia e de laboratórios de patologia, assim como a existência de serviços de referência para exames e terapias - colpocitologia e colposcopia - no município.

Embora a rede esteja organizada com serviços de referência, os profissionais de saúde da Atenção Básica apontaram diversas dificuldades no funcionamento da referência e contra-referência: número limitado de vagas para referenciar pacientes; retorno da guia de contra-referência preenchida em algumas situações (pacientes atendidas no serviço de referência), porém, na maioria das vezes a própria paciente "faz" a contra-referência, relatando, no retorno, o atendimento que recebeu. Os profissionais da Atenção Básica afirmaram que as unidades hospitalares nunca realizam a contra-referência. Os profissionais do serviço de referência queixaram-se de que muitas referências são desnecessárias, pois são casos que poderiam ser tratados na unidade que referenciou. Portanto, apesar do município organizar uma rede de referência para níveis de maior complexidade, há falhas na garantia de atendimento às usuárias. Dessa forma, a integralidade da atenção à saúde apresenta-se comprometida.

Em relação à coleta de colpocitologia, o percentual de unidades que oferecem o serviço foi alto. Vale lembrar que dentre as unidades de Atenção Básica investigadas incluíam-se unidades de saúde da família, e o médico, dentro desta especialidade, também realizava a coleta de material para o preventivo. As usuárias identificam que é o médico de família o profissional responsável pelo encaminhamento para outros níveis de atenção, assim como identificam a realização da busca ativa por este profissional.

Os indicadores qualitativos de integralidade denotam uma maior valorização pelas práticas de promoção à saúde pelos profissionais, fruto de uma visão ampliada sobre saúde, conforme apontado pelos mesmos no GF. No entanto, os profissionais do serviço de referência relataram realizar as ações de promoção ainda de forma individualizada, não se identificando ações intersetoriais para o processo de educação em saúde.

As usuárias ainda mantêm a visão limitada da saúde como ausência da doença, mas demonstram ter conhecimento acerca do câncer cérvico-uterino. Isso pode ser resultante do trabalho de promoção à saúde executado pelos profissionais, já que eles demonstraram valorizar esta prática.

As condições de registros nos estabelecimentos sugerem deficiências na continuidade da assistência, refletindo baixa qualidade técnico-científica. A importância da continuidade da assistência reside no fato de possibilitar a outro profissional da equipe de saúde, que atenda a um determinado usuário, conhecer as condutas tomadas pelos profissionais em atendimentos anteriores, assim como conhecer o histórico do usuário. Mostra o dia-a-dia dos atendimentos e o grau de importância dado pelo profissional a esse aspecto assistencial. Donabedian 13 considera os registros médicos como ponto chave para informar acerca do processo e seu resultado imediato; além disso, evidencia a qualidade do atendimento.

No grupo focal os profissionais do serviço de referência informaram realizar o registro dos atendimentos em prontuários, em estatísticas de biopsia e de citologia. Relataram ainda possuir um revisor de prontuário. Parece, contudo, que esse é ainda um trabalho mecânico, não sendo integrado ao ato de programar e executar as ações no nível local por meio de reuniões de equipe, análises clínicas de prontuário etc., que 
evidenciam o limite da capacidade técnica/qualidade da atenção.

A taxa de mortalidade apresentada pelo município em estudo no ano de 2002 (5,47 para 100 mil mulheres), encontra-se acima dos valores médios verificados no período de 1995-1999 para o Estado do Rio de Janeiro, que foram de 5,27 (taxa padronizada para a população mundial) e de 4,36 (taxa padronizada para a população brasileira) 23 . Tal resultado sugere dificuldades na implementação do programa, e conseqüentemente no controle da doença.

A condição marcadora "câncer cérvico-uterino" permitiu analisar todos os atributos da Atenção Básica selecionados: acessibilidade reduzida; falta de capacidade de planejamento, programação e gerência pela ausência do uso de indicadores de cobertura da população adscrita; deficiências do sistema que impedem a integralidade das ações, pois embora a rede se estruture numa tentativa de manter a integralidade, por meio de serviços de referência para níveis mais complexos de atenção, ficou evidenciado pelos dados do GF que existem muitas limitações; baixa qualidade-técnico-científica. Portanto, mulheres que apresentam alteração no resultado de seus preventivos ginecológicos encontram dificuldades em obter o tratamento adequado, o que possibilita a doença atingir estágios mais avançados, justificando a taxa de mortalidade encontrada como resultado.

Ao se avaliar estrutura, processo e resultado através da condição marcadora, verifica-se que os indicadores, quando analisados em conjunto, conduziram a um julgamento a respeito da Atenção Básica. Os dados qualitativos contribuíram muito para elucidar esses pontos, pois através das falas dos atores envolvidos, evidenciaramse os fatos facilitadores e as dificuldades para o funcionamento do sistema.

\section{Considerações finais}

O pressuposto de que a condição marcadora escolhida era adequada ao objetivo por suas características institucionais e de normatização assistencial se confirmou. Analisando-se o conjunto de indicadores de estrutura e processo, verificase que a condição marcadora permitiu detectar a existência de deficiências na Atenção Básica prestada no Município 3.

A proposta de uso do câncer cérvico-uterino como condição marcadora mostrou-se satisfatória. Foi possível analisar o acesso e o fluxo das usuárias dentro do sistema de saúde, identificando pontos chave que apresentam falhas na Atenção Básica. A construção da matriz permitiu organizar os atributos da Atenção Básica, possibilitando relacioná-los aos indicadores de estrutura, processo e resultado trabalhados no estudo. Essa relação entre os atributos e os indicadores possibilitou afirmar que existem deficiências na Atenção Básica que comprometem a acessibilidade, cobertura e adscrição, integralidade, continuidade técnico-científica e efetividade.

O estudo apresentou como limitação a falta de dados a respeito do seguimento das usuárias portadoras de câncer cérvico-uterino, pois não foi trabalhada nenhuma questão envolvendo o tema num dos cinco instrumentos do ELBPROESF.

A avaliação a partir do uso de condição marcadora é um método de baixo custo. O método de triangulação, que se utiliza de diversos instrumentos, mostrou a importância do aspecto qualitativo para uma melhor compreensão da realidade. Na prática, a avaliação a partir do uso de condições marcadoras poderia promover debates com profissionais e usuários para uma melhor análise dos dados quantitativos já produzidos. 


\section{Resumo}

O uso de condições marcadoras/traçadoras como técnica avaliativa sugere a possibilidade de inferência da qualidade da atenção à saúde e definição de focos avaliativos das ações programáticas. A Atenção Básica, enquanto estratégia governamental, constitui importante elemento de reorganização do modelo as sistencial. Este estudo objetiva refletir sobre o uso da condição marcadora "câncer cérvico-uterino" como mecanismo de avaliação da Atenção Básica. Com base nos resultados obtidos pelas questões relativas às ações voltadas para o controle da doença, da percepção de profissionais de saúde e de usuárias portadoras da patologia, construiu-se uma matriz de indicadores relacionando atributos da Atenção Básica e indicadores de processo/resultado. O plano de análise utilizado para validar os resultados fez uso da triangulação de métodos, associando dados provenientes das abordagens qualitativa e quantitativa, além de combinar e cruzar diversos pontos de vista apreendidos dos atores envolvidos. Os resultados encontrados sugerem o câncer de colo uterino como condição marcadora da Atenção Básica por excelência, a partir da similaridade de conceitos como acessibilidade, cobertura, integralidade, qualidade técnico-científica e efetividade.

Atenção Primária à Saúde; Avaliação em Saúde; Neoplasias Uterinas

\section{Referências}

1. Novaes HMD. Pesquisa em, sobre e para os serviços de saúde: panorama internacional e questões para a pesquisa em saúde no Brasil. Cad Saúde Pública 2004; 20 Suppl 2:S147-73.

2. Lopes RM, Vieira-da-Silva, LM, Hartz ZMA. Teste de uma metodologia para avaliar a organização, acesso e qualidade técnica do cuidado na atenção à diarréia na infância. Cad Saúde Pública 2004; 20 Suppl 2:S283-97.

3. Kessner DM, Kalk CE, Singer J. Assessing health quality - the case for tracers. N Engl J Med 1973; 288:189-94.

4. Travassos CMR. Tracer studies - a discussion of the method and of its application to health care evaluation in Brazil [Dissertação de Mestrado]. London: London School of Hygiene and Tropical Medicine; 1985.

5. Penna MLF. Condição marcadora e evento sentinela na avaliação de serviços de saúde. In: Santana JP, organizadores. Desenvolvimento gerencial de unidades básicas do Sistema Único de Saúde. Brasília: Organização Pan-Americana da Saúde/Organização Mundial da Saúde; 1997.

6. Hartz ZMA, Contandriopoulos AP. Integralidade da atenção e integração de serviços de saúde: desafios para avaliar a implantação de um "sistema sem muros”. Cad Saúde Pública 2004; 20 Suppl 2: S331-6.

\section{Colaboradores}

C. M. S. Bottari e M. M. Vasconcellos contribuíram com o desenho do estudo, a coordenação do trabalho de campo, análise dos dados, discussão dos resultados e redação do texto. M. H. M. Mendonça contribuiu na análise dos dados, discussão dos resultados e redação do texto.
7. Aleixo Neto A. Aspectos epidemiológicos do câncer cervical. Rev Saúde Pública 1991; 25:326-33.

8. Bicalho SM, Aleixo JLM. O Programa "Viva Mulher": programa nacional de controle do câncer de colo uterino e de mama. Revista Mineira de Saúde Pública 2002; (1). http://www.esp.mg.gov.br/ comunicacao/imagens_comunicacao/revista/ 1\%20-\%20Programa\%20Viva\%20Mulher.pdf .

9. Coordenação de Prevenção e Vigilância. Estimativa 2006: incidência de câncer no Brasil. Rio de Janeiro: Instituto Nacional de Câncer, Ministério da Saúde; 2005.

10. Samico I, Hartz ZMA, Felisberto E, Frias PG. A sala de situação na unidade de saúde da família: o Sistema de Informação de Atenção Básica (SIAB) como instrumento para o planejamento estratégico local. Saúde Debate 2002; 26:236-44.

11. Mendonça MHM, Najar A, Giovanella L, Tavares MFL, Vasconcellos MM, Baptista TWF, et al. Relatório síntese estudo de linha de base em 22 Municípios de mais de cem mil habitantes no Estado do Rio de Janeiro. Rio de Janeiro: Escola Nacional de Saúde Pública, Fundação Oswaldo Cruz; 2006. 
12. Minayo MCS. Introdução: conceito de avaliação por triangulação de métodos. In: Minayo MCS, Assis SG, Souza ER, organizadores. Avaliação por triangulação de métodos: abordagem de programas sociais. Rio de Janeiro: Editora Fiocruz; 2005. p. 19-51.

13. Donabedian A. The quality of care. How can it be assessed? JAMA 1988; 260:1743-8.

14. Silva LMV. Conceitos, abordagens e estratégias para a avaliação em saúde. In: Hartz ZMA, Silva LMV, organizadores. Avaliação em saúde: dos modelos teóricos à prática na avaliação de programas e sistemas de saúde. Salvador: Editora da UFBA/Rio de Janeiro: Editora Fiocruz; 2005. p. 15-39.

15. Starfield B. Atenção primária: equilíbrio entre necessidades de saúde, serviços e tecnologia. 2a Ed. Brasília: Organização das Nações Unidas para a Educação, a Ciência e a Cultura/Ministério da Saúde; 2004.

16. Secretaria de Assistência à Saúde. Manual para a organização da atenção básica. Brasília: Ministério da Saúde; 1999.

17. Mattos RA. A integralidade na prática (ou sobre a prática da integralidade). Cad Saúde Pública 2004; 20:1411-6.

18. World Health Organization. National câncer control programmes - policies and managerial guidelines. http://www.who.int/cancer/media/en/408. pdf (acessado em 10/Jan/2007).
19. Instituto Nacional do Câncer, Ministério da Saúde Viva Mulher - Programa de controle do câncer do colo do útero. Periodicidade de realização do exame preventivo do câncer do colo do útero. http://www. inca.gov.br/prevencao/programas/viva_mulher/ documentos/nota_tecnica.pdf (acessado em 11/ Out/2007).

20. Departamento de Apoio à Descentralização, Secretaria Executiva, Ministério da Saúde. Regulamento dos pactos pela vida e de gestão. http:// bvsms.saude.gov.br/bvs/publicacoes/PactosPela Vida_Vol2RegulamGestao.pdf. (acessado em 11/ Out/2007).

21. Health Canada. Programmatic guidelines for screening for cancer of the cervix in Canada. http:// www.phac-aspc.gc.ca/ccdpc-cpcmc/cc-ccu/pdf/ screening.pdf (acessado em 11/Out/2007)

22. Teixeira CF, Paim JS, Vilasbôas AL. SUS, modelos assistenciais e vigilância da saúde. Inf Epidemiol SUS 1998; 7:8-28.

23. Coordenação de Prevenção e Vigilância, Instituto Nacional do Câncer, Secretaria Nacional de Assistência à Saúde, Ministério da Saúde. Atlas de mortalidade por câncer no Brasil 1979-1999. Rio de Janeiro: Instituto Nacional de Câncer; 2002.

Recebido em 14/Mai/2007

Versão final reapresentada em 04/Out/2007 Aprovado em 08/Out/2007 